\title{
AN ELECTRON-MICROSCOPIC STUDY OF THE INFECTIVE AGENT OF TRACHOMA*
}

\author{
BY \\ YAEJI ITÔ, TAISUKE ITÔ, AND YOSHIKUNI SASAKI \\ Department of Ophthalmology, Chiba University, Japan
}

WhILE there is good reason for the belief that trachoma is due to a virus infection, the exact nature and structure of the virus is still a matter of conjecture. Attempts have been made to incriminate both bacteria and Rickettsiae as well as viruses as the cause of trachoma, but with the exception of the Halberstaedter-Prowazek bodies discovered in 1907 (Halberstaedter and Prowazek, 1910), no infective agent has been found to be constantly associated with the disease.

Since the discovery of the Halberstaedter-Prowazek bodies (H.P.K.) the most important investigations have been filtration experiments, such as those of Thygeson and others (1935) and Julianelle and Harrison (1935), which demonstrated that the infective agent was associated with a particle of about $0.25 \mu$ size. They considered that this infective particle was the elementary body seen in the H.P.K.

Neither elementary bodies nor H.P.K. can be demonstrated in all the tissues in which trachomatous changes occur. Recently a number of workers, such as Mori (1950), have again brought forward the idea that H.P.K. are purely reaction products not associated with any specific virus.

This paper reports the occurrence in trachomatous tissues of bodies, which we have provisionally named " $\mathrm{Z}$ " bodies. These bodies have been studied by electron-microscopy and have been used in infectivity experiments. We have been able to show that they are present only in trachomatous tissue and are not found in similar material from persons not suffering from trachoma.

\section{Material AND Methods}

The following tissues have been used for the isolation of " $\mathrm{Z}$ " bodies:

(1) Conjunctival washings of trachoma, Stage IIa, showing a marked inflammatory reaction.

(2) Conjunctiva excised from all sites in cases of trachoma, Stage IIa.

(3) Palpebral conjunctiva from trachoma, Stages I to III.

* Received for publication March 27, 1951. 
(4) Pannus from trachoma, Stage III.

(5) Tissue from chronic dacryocystitis associated with trachoma.

(6) Upper tarsus in trachoma, Stage III.

Conjunctival washings are made by repeatedly washing the conjunctival sac with up to $10 \mathrm{ml}$. physiological saline. Tissues are excised, ground in a mortar, and made up to $10 \mathrm{ml}$. with saline.

The material, either washings or emulsion, is brought up to $p \mathbf{H ~} 7.8$ with phosphate buffer and centrifuged at 3,000 revolutions per min. for $10 \mathrm{~min}$.

Supernatant fluid from the centrifugalization is now treated by the technique described by Hasumi (1950) for the isolation of sarcoma virus. This consists of a preliminary filtration through a column of refined asbestos $2 \mathrm{~cm}$. in height and $1 \mathrm{~cm}$. bore under a negative pressure of $20 \mathrm{~mm}$. Hg. The asbestos should be packed so that the rate of flow is 7 to 8 drops a minute. The material clarified by the preliminary filtration is drawn through a chromatographic column $20 \times 1 \mathrm{~cm}$. in size composed of active kaolin. The column is packed in a glass tube plugged at either end with absorbent cotton. Negative pressure is used to draw the material through the column.

All apparatus is steam sterilized before use.

When adsorption of material on the column is completed, the column is pushed out of its glass tube and cut into small sections. Each section

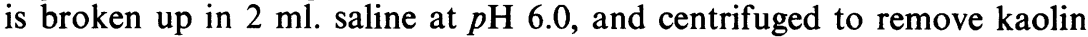
(3,000 r.p.m. for $10 \mathrm{~min}$.). After centrifuging, the supernatant fluid is used for the preparation of films for electron-microscopy.

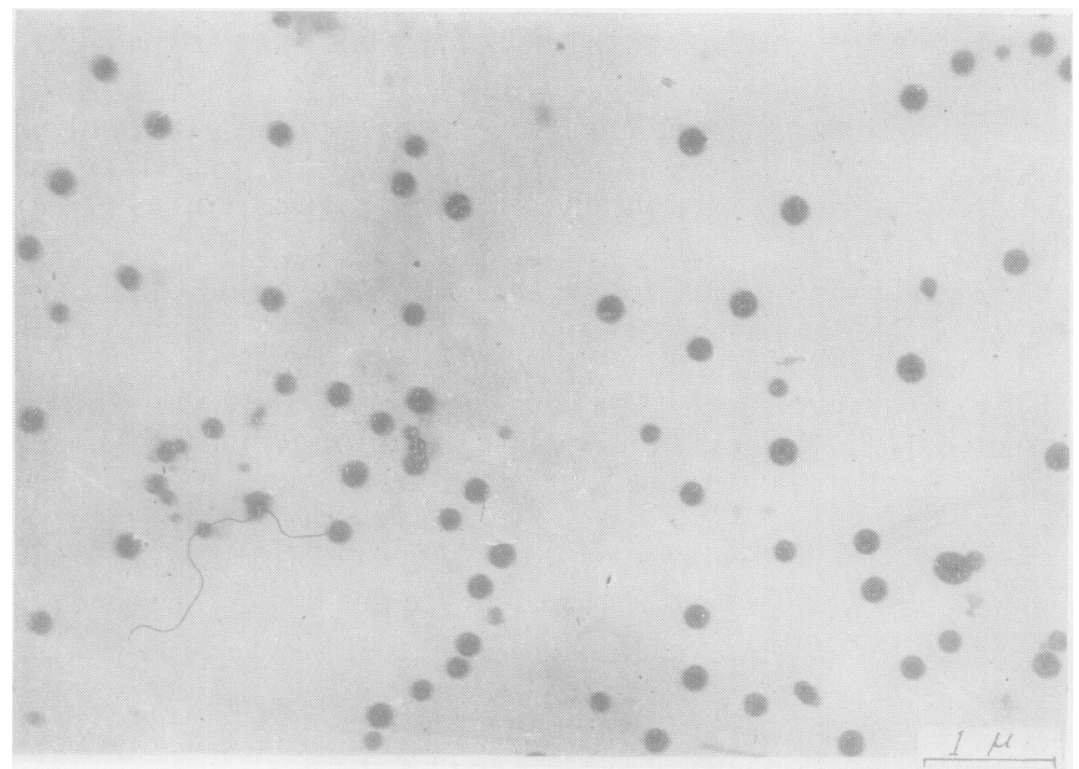

FIG. 1.-Electron-microscope photograph of " $\mathrm{Z}$ " bodies. 


\section{RESULTS}

In the first $4 \mathrm{~cm}$. of the column, round or oblong particles are found. These particles (shown in Fig. 1) are usually from 60-250 $\mathrm{m} \mu$ diameter but their range is from $20-300 \mathrm{~m} \mu$. A histogram of sizes of these particles gives the mode as lying at $180 \mathrm{~m} \mu$ (Fig. 2).

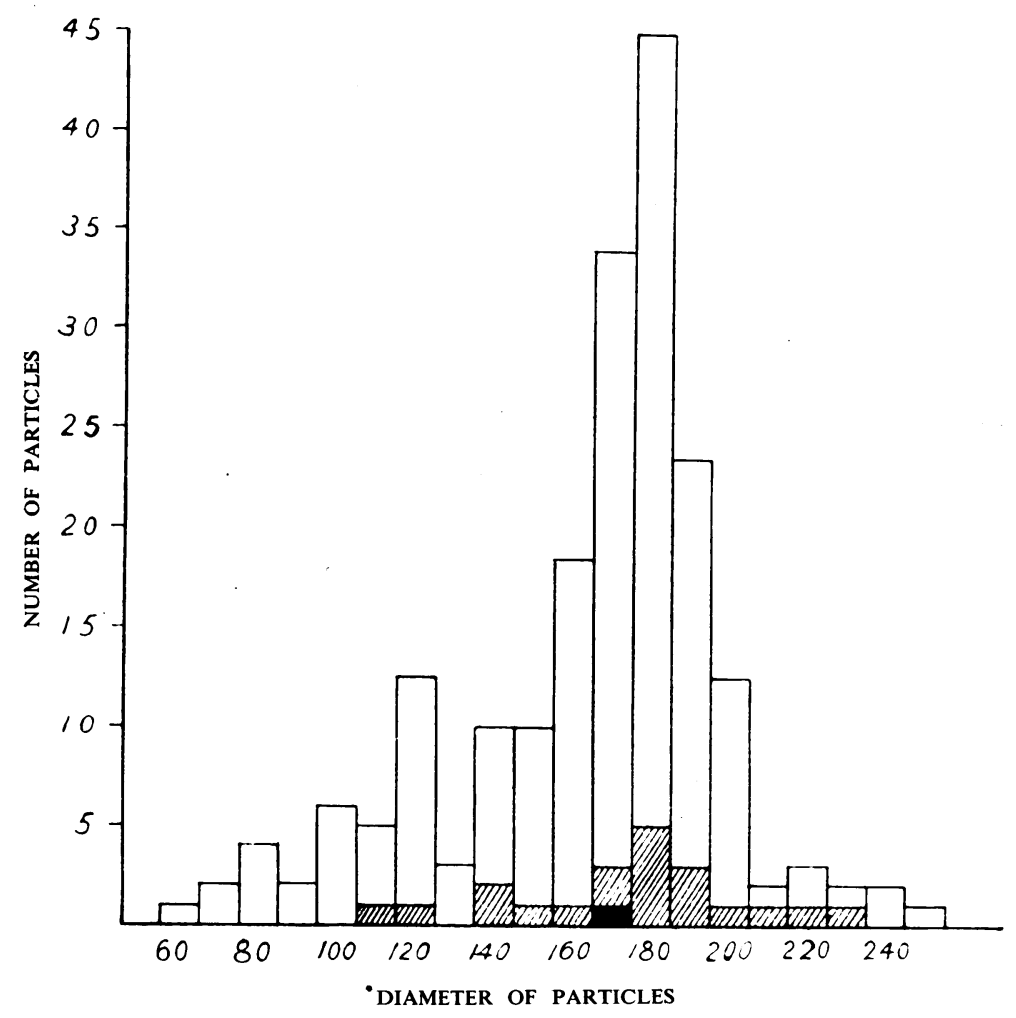

FIG. 2.-Histogram showing distribution of particle sizes

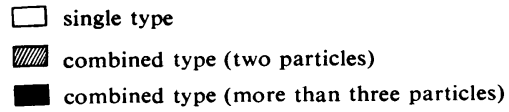

No similar particles could be found in tissues, taken from the corresponding areas, and treated by the same technique, from persons not suffering from trachoma.

The electron-micrographs show that, while most of the particles are single, several show two or three particles joined together and occasionally a single particle may have a protrusion at one point. 


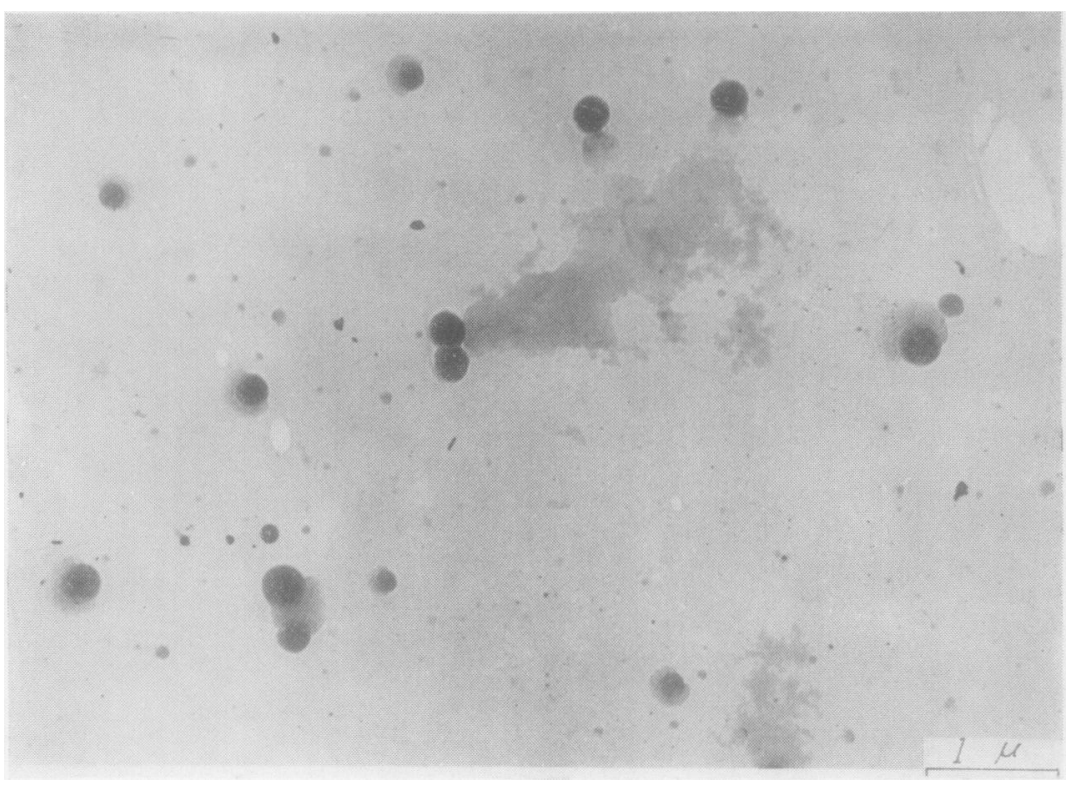

FIG. 3._" Z" bodies showing plasmoid capsule.

Fig. 3 shows the " $Z$ " bodies together with a surrounding envelope. This envelope can only be found immediately after the isolation of the bodies from the chromatographic column for they disappear if the material is allowed to stand. The bodies can be more readily seen if the kaolin is broken up in distilled water instead of buffered saline. The sizes of bodies and envelope can be estimated and averages $590 \mathrm{~m} \mu$. The envelope is similar to a structure seen around the particles of the psittacosis virus, to which group of viruses it is thought that the trachoma virus may belong.

We call the main mass of the particle the nucleoid body, and the envelope the plasmoid capsule. Plasmoid capsules can be seen around " $\mathrm{Z}$ " bodies of from $110 \mathrm{~m}: \iota$ upwards, but are best seen in those over $150 \mathrm{~m} \mu$; it seems that they increase in size proportionately with an increase in size of the nucleoid body. The smaller " $Z$ " bodies, when stained by Giemsa's stain, were eosinophilic, while the larger particles stained more intensely and were basophilic. This staining resembles that of the elementary and initial bodies of H.P.K. respectively. If the plasmoid capsule was destroyed the bodies could not be stained by Giemsa's stain.

The multiple bodies seen in the pictures of " $\mathrm{Z}$ " bodies are often composed of different-sized particles, and by measurements we estimated the ratios of their cubic volume. Using these measurements we divided the multiple bodies into four groups: 
(a) both particles the same size,

(b) smaller particle more than half as big as the larger,

(c) smaller particle less than half as big as the larger, usually with two particles combined,

(d) types in which there is a protrusion of material at some point, but no separation into two distinct particles.

It seems that these differing bodies represent stages in the multiplication of the virus; the first process is a germination to form a $(d)$ type, which then increases until the $(c)$ and $(b)$ types are formed. At some point in the development of $(c)$ and $(b)$, fission occurs to form a fresh particle. The smallest fission particle which has a free existence is about $60 \mathrm{~m} u$ diameter.

\section{TRANSMISSION EXPERIMENTS}

Five human volunteers were infected with material containing " $\mathrm{Z}$ " bodies, Case 1 with material passed through the preliminary filter only, and Cases 2-5 with material collected by the chromatographic column.

Cases 1, 2, and 3 developed trachoma, Case 4 developed trachoma only after a second inoculation, and Case 5 did not " take" after two inoculations.

The inoculum was applied in two forms; first a few drops of the suspension was placed in the conjunctival sac, and a few minutes later a suspension of the " $\mathrm{Z}$ " bodies in equal parts of anhydrous lanoline and vaseline was applied to the conjunctiva with a glass rod.

The results of inoculation may be summarized as follows:

(1) Latent Period before Symptoms.-For Cases 1 and 2 this was 4 days, and for Cases 3 and 4 it was 3 and 5 days respectively. The first sign of infection was conjunctival congestion.

(2) Subjective Symptoms.-A sensation of grittiness and conjunctival irritation was experienced from the $3 \mathrm{rd}$ or 4 th day. There was a gradual increasing serofibrinous discharge and this eventually became purulent. Photophobia only occurred if there were complications.

(3) Conjunctiva of Lids and Fornix.-Congestion started on the 3rd to the 5 th day, and between the 5 th and 10th days papillary hypertrophy developed at both inner and outer canthus. This spread diffusely over the lids between the 12th and 20th days.

(4) Pannus.-This developed on the 21st day in Cases 1 and 2, and on the 19th day in Case 4.

(5) Ptosis of the Lid.-This was seen in all cases.

(6) H.P.K.-These were first found on the 6th day in Case 4 . Case 1 did not develop them until the 13th day.

- (7) Bulbar Conjunctiva.-This displayed the trachomatous response described by Itô and Yu (1951). 
Cases were observed for varying periods, depending on the subective symptoms in the volunteers. Treatment was 10 per cent. Marfanil ointment, which brought symptomatic relief with disappearance of H.P.K.

" $Z$ " Bodies in TRANSMISSION EXPERIMENTS.- " $Z$ " bodies were demonstrated in all the material inoculated and were found in those volunteers who developed symptoms (Cases 1-4), but could not be demonstrated in Case 5 which did not develop clinical trachoma. The " $Z$ " bodies shown in Fig. 4 are from one of the volunteers.

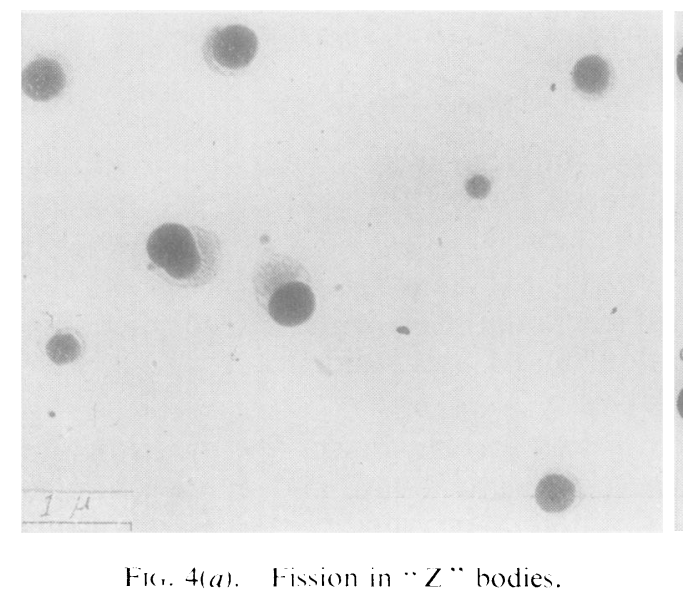

\section{Discossion}

Filtration experiments have shown that particles down to $0.25 \mu$ were infective, except in the experiments of Braley (1949),

which suggested that particles from 100-200 $\mathrm{m} \mu$ might be infective. All these experiments showed a marked drop in the infective titre of material after filtration, a finding which could be explained by inequality of particle size, and in these electron-micrographs " $\mathrm{Z}$ " bodies can be shown to vary in size. This inequality of particle size could only be demonstrated by some method of isolation, such as adsorption in a chromatographic column, which is dependent upon properties other than size for isolation. In a chromatographic column localization is due to the similarity of adsorption characteristics of the adsorbed substance, and is independent of size.

The sizes of particle isolated by our technique vary from a size suggestive of initial bodies down to $60 \mathrm{~m} \mu$ or particles of approximately one quarter the size of elementary bodies estimated by microscopy and filtration. 
A number of the particles can be shown to possess an envelope which we have called the plasmoid capsule. This may be similar to the envelope which has been shown to surround the elementary body of the psittacosis virus. Unfortunately, it has not been possible to demonstrate whether the infective nature of the material is dependent upon any single size of particle or whether the presence of a plasmoid capsule plays any part in infectivity.

Study of the electron micrographs suggest that the virus grows by germination, and that the material that buds out is separated from the parent body by fission when the daughter particle reaches a diameter of about $60 \mathrm{~m} \mu$ or greater. Groupé and Rake (1947), working with fowl pox, and Heinmets and Golub (1948), working with psittacosis, have suggested that fission in viruses may occur. We believe that the only explanation of the micrographs is that such fission occurs. Fission seems to be associated with the plasmoid capsule; if this is the case, only those particles with the plasmoid capsule could propagate, which would mean that virus multiplication would be restricted to elementary and initial bodies. This might well be so, since " $\mathrm{Z}$ " bodies are found in those cases which have H.P.K., and infection of volunteers with " $\mathrm{Z}$ " bodies leads to the formation of H.P.K. in the conjunctival epithelium.

\section{SUMMARY}

Electron-microscopy has shown a pleomorphic particle associated with tissues infected by trachoma. These particles, which have been called " $\mathrm{Z}$ " bodies, consist of a central mass, a nucleoid body, and an envelope or plasmoid capsule.

Multiplication of " $\mathrm{Z}$ " bodies is by fission.

" $\mathrm{Z}$ " bodies are capable of causing trachomatous infection in human volunteers.

\section{REFERENCES}

Braley, A. E. (1949). Arch. Ophthal., Chicago, 41, 151.

Groupé, V., and RAKe, G. (1947). J. Bact., 53, 449.

Halberstaedter, L., and Prowazek, S. V. (1910). Berl. klin. Wschr., 47, 661.

Hasumi, K. (19j0). Shizen (Nature), 5, No. 6, p. 71.

HeINMETS, F., and Golub, O. J. (1948). J. Bact., 56, 509.

ITô, Y., and YU, C.-H. (1951). British Journal of Ophthalmology, 35, 304.

Julianelle, L. A., and Harrison, R. W. (1935). Amer. J. Ophthal., 18, 133.

MORI, N. (1950). Acta Soc. ophthal. jap., 54, 362.

Thygeson, P., Proctor, F. I., and Richards, P. (1935). Amer. J. Ophthal., $18,811$. 